Pensamiento Crítico N. ${ }^{\circ}$ 11, pp. 73-96

\title{
El caserío es la unidad productiva en la articulación organizacional de zonas rurales altoandinas, Callejón de Huaylas, Ancash
}

Mildred Paredes Tarazona

\section{RESUMEN}

El objeto es precisar tres indicadores significativos identificados para la articulación organizacional en zonas rurales altoandinas sustentados en la psicología económica; los estudios se realizaron en los caseríos Queral, Miramar, Chinchay, Pampacocha de la Comunidad Campesina Cruz de Mayo, desde 2003, en población mayor de 16 años; los resultados señalan que la variable caserío discrimina con mayor eficacia entre las dimensiones psicosociales de su construcción social de la organización para su representación social organizacional de la comunidad campesina; de igual manera, es aquella que produce sinergia por la percepción de sus integrantes hacia el conocimiento de la demanda para formar interdependencias económicas entre caseríos, y discrimina la percepción de las condiciones de trabajo otorgando la competencia del manejo del medio ambiente a la Comunidad. Se han empleado escalas de percepciones válidas y confiables para cada investigación y los actores de cada unidad económica 


\title{
Mildred Paredes Tarazona
}

de consumo fueron encuestados en quechua de la zona por un representante designado de la comunidad.

Palabras clave: Productiva, caseríos, sinergia, percepción, trabajo.

\begin{abstract}
The object is to specify three significant indicators identified for the joint organizational highland rural areas supported by economic psychology, and studies were conducted in the villages Queral, Miramar, Chinchay, the Rural Community Pampacocha Cruz de Mayo, since 2003, population over 16 years, the results indicate that hamlet variable discriminates more effectively between the psychosocial dimensions of their social construction of social organization for organizational representation of the peasant community, likewise, is one that produces synergy by the perception its members to the knowledge of the demand to form economic interdependencies between villages, and discriminates against the perception of working conditions of the competition by providing environmental management to the Community. It has been used perceptions scales valid and reliable for each research and economic actors of each consumer unit were surveyed in Quechua in the area by a designated representative of the Community.
\end{abstract}

Keywords: Productive, farmhouses, synergy, perception, work.

\section{INTRODUCCIÓN}

Los resultados significativos obtenidos de los estudios orientados a conseguir información válida y confiable correspondiente a la percepción de factores de cohesión predominantes de la dimensión psicosocial o subjetiva de la organización productiva altoandinas, cuya imagen construida por los pobladores de las zonas rurales, son presentadas a la comunidad académica e interesados, con el propósito de ser transferidos a organizaciones o instituciones cuya competencia es la generación de programas de intervención social dentro los lineamientos de la gestión de calidad y promotora de una economía sostenible que esté de acorde a su cultura y saberes.

Los requerimientos son explícitos en toda agenda de un programa de intervención, pero muchas veces soslayan aquellas demandas de sus propios actores, especialmente si se repara en la calidad humana que proporciona la calidad de vida expresada en 

andinas, Callejón de Huaylas, Ancash

preservar la vida de dicha población con salud en toda actividad como el trabajo, estudio y juego según el respeto y valoración a su diversidad cultural; en tal sentido, entre los objetivos de interés académico, está el generar el conocimiento de dicha realidad sui géneris, calificada e identificada por la representación de la construcción social de las comunidades campesinas andinas otorgada por sus propios actores sociales; información válida para decisiones; en especial la decisión económica, que se sustenta en la igualdad de valor asignado a los recursos tangibles como a los psicológicos y de riqueza cultural que vincula su crecimiento económico por sus competencias y pericias.

El foco de interés para el estudio son cuatro caseríos, y el periodo data del 2003: Pampacocha, Queral, Chinchay y Miramar pertenecientes a la Comunidad Campesina Cruz de Mayo, ubicadas a las faldas del la Cordillera Blanca; Caraz, Huaylas, Ancah (ver anexo N. ${ }^{\circ}$ ); los datos se obtuvieron mediante la técnicas ad hoc validadas y confiables a cada estudio (escalas de percepción y actitudes, registro de la autopercepción y una escala de cotejo), resueltas por los integrantes de cada caserío de la comunidad. La data se procesó según el paquete estadístico SPSS versión 12 con técnicas estadísticas no paramétricas.

Tabla N. ${ }^{\circ}$ 1. Población del estudio.

\begin{tabular}{|c|c|c|c|c|c|c|c|c|c|c|}
\hline \multirow[t]{2}{*}{ Caserío } & \multirow[t]{2}{*}{ Sujetos } & \multirow[t]{2}{*}{ Familias } & \multirow{2}{*}{$\begin{array}{l}\text { Mayores } \\
\text { de } 16 \\
\text { años }\end{array}$} & \multirow{2}{*}{\multicolumn{3}{|c|}{$\begin{array}{r}\text { Muestra } \\
\mathbf{e}=0,05\end{array}$}} & \multirow{2}{*}{\multicolumn{3}{|c|}{$\begin{array}{c}\text { Muestra } \\
\mathbf{e}=\mathbf{0 , 1}\end{array}$}} & $\begin{array}{c}\text { Muestra } \\
e=0,1\end{array}$ \\
\hline & & & & & & & & & & Real \\
\hline \multirow{2}{*}{ Pampacocha } & \multirow{2}{*}{509} & \multirow{2}{*}{85} & \multirow{2}{*}{254} & \multirow{2}{*}{103} & 51 & M & \multirow{2}{*}{38} & 19 & M & \multirow{2}{*}{42} \\
\hline & & & & & 52 & V & & 19 & $\mathrm{~V}$ & \\
\hline \multirow{2}{*}{ Queral } & \multirow{2}{*}{241} & \multirow{2}{*}{50} & \multirow{2}{*}{125} & \multirow{2}{*}{52} & 28 & M & \multirow{2}{*}{19} & 10 & M & \multirow{2}{*}{34} \\
\hline & & & & & 24 & V & & 9 & $\mathrm{~V}$ & \\
\hline \multirow{2}{*}{ Miramar } & \multirow{2}{*}{208} & \multirow{2}{*}{43} & \multirow{2}{*}{106} & \multirow{2}{*}{44} & 23 & $M$ & \multirow{2}{*}{16} & 8 & $M$ & \multirow{2}{*}{6} \\
\hline & & & & & 21 & $\mathrm{~V}$ & & 8 & V & \\
\hline \multirow{2}{*}{ Chinchay } & \multirow{2}{*}{117} & \multirow{2}{*}{16} & \multirow{2}{*}{61} & \multirow{2}{*}{25} & 13 & $M$ & \multirow{2}{*}{9} & 5 & $M$ & \multirow{2}{*}{16} \\
\hline & & & & & 12 & $\mathrm{~V}$ & & 4 & $\mathrm{~V}$ & \\
\hline \multirow{2}{*}{ Totales } & \multirow{2}{*}{1075} & \multirow{2}{*}{194} & \multirow{2}{*}{546} & & 116 & M & & 42 & M & 0 \\
\hline & & & & 224 & 108 & $\mathrm{~V}$ & 82 & 40 & $\mathrm{~V}$ & 98 \\
\hline
\end{tabular}

Fuente: Posta de salud de Pampacocha (Paredes, M. \& De Miguel, J., 2004). 


\section{Mildred Paredes Tarazona}

Por los resultados, se puede confirmar que la variable caserío es aquella que agrupa y posibilita la discriminación con mayor poder de imagen que los miembros tienen de su comunidad (Paredes, M. y De Miguel, Jesús 2004); la misma variable también presenta resultados similares al describirse cómo se presentan las percepciones de las condiciones de trabajo y el manejo del medio ambiente (Paredes, M., De Miguel, J., Hernández, H., Vicuña, L., y colaboradores 2005).

En la población total se observa que hay relación significativa entre las condiciones de trabajo y el manejo del medio ambiente, según Pearson $(0,422)$, destacándose marcadamente que se diferencia la percepción a las condiciones de trabajo por caserío, dada la existencia de una construcción social única de cada caserío. No se diferencia así en cuanto al manejo del medio ambiente, lo cual atribuye competencia a la construcción social de la organización de la comunidad.

De tales datos se puede inferir que el papel o el rol de la construcción social de la organización de la comunidad tiene incidencia en la percepción de sus miembros hacia las condiciones de trabajo y el manejo del medio; de ahí que la Psicología Social Organizacional, como ciencia aplicada a las organizaciones, busca la prevención de la salud de los miembros o integrantes de la organización para lograr fortalecer su desarrollo óptimo; en tal sentido, observa, describe, explica e interviene, con efectividad sustentada entre las dimensiones psicosociales, la percepción de la realidad que construyen sus integrantes; tal variable psicológica cimenta la imagen para la promoción y fortalecimiento de la conducta saludable observada según el tipo de interrelación que ha aprendido la persona-organización-medio y la interrelación medio- organización-persona; es punto de partida para la búsqueda de coadyuvar tanto a la salud (la misma se entiende como una situación de calidad de vida del individuo y de su comunidad en interrelación con su medio) como para el desarrollo local mediante la autoidentificación y desarrollo de proyectos productivos competitivos que sumen al progreso económico sostenible.

En este menester, es necesario precisar que la percepción de la situación de calidad de vida en interrelación con el medio ambiente y preservación de la ecología es el indicador de bienestar y desarrollo económico sostenible, no solo de una organización, sino también de la sociedad como un factor básico en la articulación organizacional. En esta vía, la autopercepción de los integrantes de una organización sobre las condiciones 
El caserío es la unidad productiva en la articulación organizacional de zonas rurales alto andinas, Callejón de Huaylas, Ancash

Tabla N. ${ }^{\circ}$ 2. Anova global y por áreas de condiciones de trabajo y manejo del medio ambiente según variables demográficas.

\begin{tabular}{|l|c|c|c|c|c|c|c|c|}
\hline \multicolumn{1}{|c|}{ VARIABLE } & $\begin{array}{c}\text { Sig. } \\
\text { Cargo }\end{array}$ & $\begin{array}{c}\text { Sig. } \\
\text { Caserío }\end{array}$ & $\begin{array}{c}\text { Sig. } \\
\text { Est. } \\
\text { Civil }\end{array}$ & $\begin{array}{c}\text { Sig. } \\
\text { Membresía }\end{array}$ & $\begin{array}{c}\text { Sig. } \\
\text { Edad }\end{array}$ & $\begin{array}{c}\text { Sig. } \\
\text { Estudio }\end{array}$ & $\begin{array}{c}\text { Sig. } \\
\text { Sexo }\end{array}$ & $\begin{array}{c}\text { No } \\
\text { Difs. }\end{array}$ \\
\hline Condiciones trabajo total & .630 & .008 & .0174 & .761 & .441 & .391 & .548 & 4 \\
\hline Condición medio físico & .696 & .814 & .658 & .010 & .505 & .479 & .295 & 3 \\
\hline Condición de salud mental & .997 & .251 & .323 & .685 & .368 & .065 & .239 & 5 \\
\hline Condición situación social & .977 & .450 & .092 & .537 & .394 & .990 & .379 & 4 \\
\hline Condición del Estado salud & .139 & .021 & .026 & .618 & .222 & .885 & .264 & 5 \\
\hline Prevención accidentes & .622 & .037 & .249 & .936 & .402 & .181 & .233 & 5 \\
\hline Factores adversos & .541 & .003 & .019 & .224 & .225 & .623 & .978 & 4 \\
\hline Factores para trabajo & .336 & .069 & .696 & .175 & .581 & .832 & .380 & 4 \\
\hline Instrumentos y equipos & .788 & .273 & .302 & .163 & .524 & .722 & .069 & 4 \\
\hline Manejo medioambiente total & .243 & .701 & .955 & .266 & .076 & .857 & .995 & 3 \\
\hline Número diferencias & 3 & 8 & 7 & 6 & 8 & 5 & 7 & \\
\hline
\end{tabular}

Difference significant al $0.5=<$ al 0.499

de trabajo no solo es necesaria para su seguridad ocupacional, la que es fundamental, sino para dar paso a vivir y convivir dentro de niveles de calidad especificados por los indicadores de organismos supranacionales y empresas abocadas al mejoramiento de la calidad de vida de la Tierra.

Si bien las condiciones de trabajo son aquellos elementos que se sitúan en torno al trabajo sin ser el trabajo mismo, el conjunto de factores que lo envuelven como tales son innumerables, además de involucrar el término de medio ambiente (Ramos, J.; Peiró José Ma. \& Ripio, P. 1996). Se tiene, entre las categorías de la taxonomía que han sistematizado dichos autores, las siguientes: condiciones del empleo; condiciones ambientales; condiciones de seguridad; características de la tarea; procesos de trabajo y condiciones sociales y organizacionales.

De los múltiples y diversos factores de las condiciones ambientales se hace necesario precisar los tres aspectos diferenciados indicados por los autores, referidos a: a) ambiente físico, la temperatura, humedad, nivel de ruido, iluminación, ventilación y pu- 


\section{Mildred Paredes Tarazona}

reza del aire, vibraciones, los aspectos electromagnéticos preferentemente; b) Variables espacio-geográficas, área y distribución de ese espacio, su configuración y las relaciones que se establecen entre el espacio y las personas; c) diseño espacial-arquitectónico, construcción en el espacio como el uso de materiales y equipamiento según los estándares identificados y recomendados por la ergonometría.

La percepción del ambiente implica actos intencionales donde el ambiente envía mensajes que ayudan al individuo a orientar sus acciones; para ello, los diversos estímulos son organizados por los procesos preceptuales para formar un cuadro coherente e integrado del mundo (Holahan, Ch. 1996, 66). Ahora bien, este ambiente puede ser físico, espacial como psicológico y socioeconómico cultural, donde se elabora o ejecuta una actividad productiva, la misma que se constituye en base fundamental para la formación de la personalidad (Rubinstein, J.L. 1967:617-665), así como el fortalecimiento del sentimiento de comunidad señalado por Adler, A. como el requisito de toda convivencia social, puesto que es más que un sentimiento, es una forma de vida, como "ver con los ojos del otro, oír con los oídos de otro, sentir con el corazón de otro" (Titze, M. 1983: 189-141). Lo que se observa, por ejemplo, cuando los integrantes de una organización, sea cual fuere su categoría o nivel ocupacional, se percatan del conocimiento de cómo realizan su labor y evitan las condiciones "sub estándar" -aquellas condiciones que de no corregirse podrían causar un accidente-, y es la partida al cambio; con darse cuenta de ello e incorporarlo al sentimiento se logra implantar la conducta, al menos en principio, y ya este tomar conocimiento va construyendo las categorías que dan significación, condición válida para el comportamiento.

La imagen de una organización se construye según la percepción de los miembros como por la de sus usuarios y consumidores; por este proceso de feedback otorgado por el rol de agente económico en toda comunidad o sociedad. Así, toda organización sea empresarial o no, desempeña un rol en la comunidad; por un lado da aquella imagen que despliega en tanto partícipe e integrante del medio ambiente signado como entorno, y por otro genera una imagen elaborada por sus integrantes y consumidores cuya imagen se va construyendo según los lazos que se establecen a nivel de la intra y de la interacción de la organización con su entorno inmediato (la familia y amigosvecinos). Esta percepción de la interacción de la organización con su medio es otro factor válido que prevalece como eje central en y para la preservación del ambiente y medio ecológico -legado de la generación actual y venidera-, sobre todo cuando nutren 


\section{El caserío es la unidad productiva en la articulación organizacional de zonas rurales alto}

andinas, Callejón de Huaylas, Ancash

o generan conductas organizacionales como la productividad, satisfacción e innovación, facilitadas por cooperación, participación e identificación.

El producto de la acción mutua es valorada y evaluada a partir de la percepción, constituyéndose en un referente a ser diseñado como indicador del manejo del medio ambiente de la organización con su entorno, tanto por la significación a partir de la categorización como por la cognición socialmente compartida, cuya perspectiva es el estar interesado en analizar la actividad que realizan en colaboración entre miembros quienes llevan conjuntamente, y no por separado, la codificación, la interpretación, el almacenamiento y el recuerdo de la información relevante para la ejecución de la tarea (Alcover de La Hera, C. M. 2000,300), en micro contextos o en grupos.

Entre los antecedentes se tiene el estudio realizado en la Comunidad Campesina Cruz de Mayo y la Comunidad Campesina de Cajabamba Alta, Caraz, Huaylas (Paredes, M. y De Miguel, J. 2004, 81-102) que califica "que cada comunidad construye y comparte una representación de la comunidad sui generis".

Los resultados presentados permiten afirmar que la construcción social organizacional de la comunidad andina vincula simbólica y emocionalmente, desde una perspectiva institucional, a la comunidad y se impone a sus miembros con la identidad; $y$, desde una perspectiva a lo institucional, por la diversidad de sus integrantes que como individuos de la comunidad ejercitan un juego simbólico que genera un conocimiento sobre la propia comunidad, (De Miguel, J.) en su modelo "Lo local como organización". Estas dimensiones psicosociales caracterizan al sistema simbólico socialmente compartido, como aquellas descritas y requeridas para la gestión de calidad bajo una óptica de sostenibilidad, calidad trastocada cuando se le compara con los indicadores de bienestar económico y peor aún cuando se observa que en sus caseríos, y como tal fundamentalmente sus actividades productivas, le proveen una economía de subsistencia a nivel precario cuando menos, con déficit en la dieta alimenticia por señalar esta necesidad perentoria que es el alimento. Esta problemática enrumba con una serie de preguntas y otras aún sin formularse a buscar respuestas científicas y tecnológicas a ser plasmadas en un diseño estratégico compatible con su realidad y la dignidad de personas y comunidad, ya en el siglo XXI de vuelta a la humanidad por privilegio. 


\section{Mildred Paredes Tarazona}

Un sistema ideal de organización se denominada $\mathrm{K}$, que en resumen se caracteriza porque considera fundamental la participación de sus integrantes tanto en las decisiones que afectan al común de la organización como aquellas que afectan al trabajo autónomo; de igual manera identifica al trabajador del conocimiento, como también su alta participación en la construcción del conocimiento de la organización. Es decir, la participación de los miembros de la organización en la generación de conocimiento sobre la propia organización y la generación y mejora del conocimiento.

Cada caserío tiene su propio clima psicosocial como resultante de su sistema social, siendo el caserío la variable que más discrimina y agrupa para diferenciar la imagen que tienen los miembros de la comunidad; imagen que así expresa sus expectativas, las que van dirigidas a lograr el resultado competitivo de su actividad laboral, estrategia sentida. al precisar, fortalecer o desarrollar sus modos y tecnología de producción, y que es la vía para que pueda su comunidad albergar y mantener el contexto psicosocial favorable para desarrollarla con el crecimiento económico según toda aspiración humana; y que sus niños hoy, jóvenes mañana, potenciales emigrantes a la urbe (hecho probable de realizarse), empobrezca cada vez más a la comunidad y perturbe sus estilos de vida con los casos que al retornar traen consigo conductas antisociales generadoras de malestar: "nos duele ver que nuestros jóvenes se pierdan", "vengan a robar, no tan solo los abigeos", "los hombres o mujeres tomen veneno", "no nos alcanza para comer menos para sus estudios, ..." entre las expresiones que buscan enfrentar y superar.

Por ello, sí se repara con cuidado en la representación social organizacional de una comunidad andina en el Perú, donde se aprecia cómo sus actores categorizan las dimensiones psicosociales en la representación social de su construcción organizacional con aquellas variables de gestión: factor común entre las variables encontradas en la escala de cognición son aquellas que en las organizaciones empresariales dominan y controlan los gerentes para propiciar equipos eficaces de producción, (Paredes, M. y De Miguel, J. 2004), graficando a continuación la escala de cognición, donde priorizan la producción por ejemplo. 

andinas, Callejón de Huaylas, Ancash

Resultados de la escala de cognición.

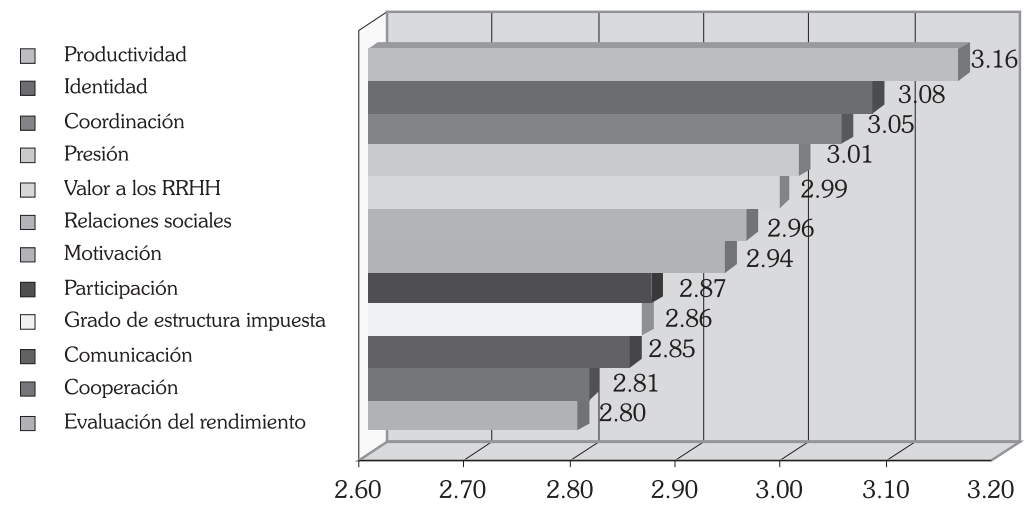

Se confirma que la intervención de las instituciones foráneas en ella debe hacerse de organización a organización, y tratarla como tal (Corbera, J.; 2008) al describir la intervención en zonas rurales andinas preferentemente mediante el instrumento "unidad focal" sustentado y fundamentado en los proyectos desarrollados por FONCODES entre 1990-1996 con el objetivo de superar la pobreza; asimismo, incluir indicadores psicológicos a fin de desvirtuar los entredichos manejados por ejemplo (C. Trivelli y C. Aramburú 2000, 20) cuando describe que "una buena parte de los pequeños productores de las zonas rurales del país que por -su idiosincrasia- propia afectan su relación con la sociedad nacional de la que forman parte, que hace difícil una interlocución bidireccional fluida con agentes del exterior y que, por ende, comprometen la aplicación de políticas de desarrollo. Por otro lado, muchas veces sus formas tradicionales de organización representan valores y estilos institucionales poco favorables para el desarrollo rural". Estas opiniones llevan a reconocer la preponderancia con que (Popper Karl R. 1997) se precisa sobre la responsabilidad moral de los científicos y la deuda con la civilización en relación al aumento del conocimiento científico y su papel que desempeña en ella, suscitando la recomendación, que es más ético asesinar o destruir el error del conocimiento, que él haga daño a las personas o a la civilización.

La mayor información sobre los aspectos de los procesos psicológicos implicados en el comportamiento económico pero igualmente cómo la economía afecta el comportamiento de los individuos (Alejo, R. Argemiro y colaboradores, 2008), como 


\section{Mildred Paredes Tarazona}

lo propugnara ya hacia 1950 Katona, G. (1963) que las actitudes, motivos de las personas y las referencias, componen el conocimiento de su medio ambiente, así como de su comportamiento; y para comprender los procesos económicos, así como otras manifestaciones de la conducta, "debe estudiarse también la variable subjetiva"; cuyo contenido psicológico se convierte en un punto de apoyo a la investigación interdisciplinaria en sus aspectos básicos y aplicados que la ecología requiere para contribuir a la construcción de una imagen más racional del vínculo de los seres humanos con la naturaleza" (Rivera, J.C. 2000) para evitar que toda intervención no solo perturbe y le acarree pérdidas económicas y de salud a sus actores.

Describir, a partir del diagnóstico de la percepción del conocimiento que tienen los miembros de la organización sobre las condiciones de trabajo y su manejo del medio ambiente, proporciona información a la gestión de la organización para fortalecer o modificar su comportamiento mediante programas acorde a su realidad tomando en cuenta la representación social organizacional peculiar de la Comunidad Campesina andina de influencia en Caraz, Ancash. La construcción creativa, según la representación social de su organización, difiere de comunidad a comunidad como de caserío a caserío, tanto en su sistema simbólico compartido a nivel imagen como actitudinal; tal característica que lleva a preguntar si guarda relación entre las condiciones de trabajo y el manejo del medio ambiente según caserío en la Comunidad Campesina de Cruz de Mayo, en especial si se ha descrito que una de las dimensiones psicosociales, la productividad, es prioritaria en la imagen y en la actitud de la representación organizacional de los miembros de la comunidad, posición en esta última antecedida por la motivación en el trabajo, identidad y relaciones humanas.

Los resultados confirman que por caserío difiere la percepción de las condiciones de trabajo, pues Queral es el caserío que reporta una percepción baja en relación con dichas condiciones de trabajo; por lo contrario es evidente que la percepción hacia el manejo del medio ambiente no difiere por caserío, y se asume que esta responsabilidad es otorgada a la Comunidad.

Por otro lado, se tiene que hay relación significativa positiva entre la percepción del conocimiento de la demanda del área de la percepción de la oferta con la interdependencia económica hacia la percepción de la oferta; el único indicador positivo significativo para lograr alianzas económicas con el propósito de orientar una gestión competitiva a 
un mediano plazo (Paredes, M y colaboradores, 2007-122); en base a la significación estadística 0,05 correlación Coeficiente Tau de Kendall, solo se presenta en la percepción del conocimiento a la oferta de los productos de su localidad con la percepción hacia a la formación de interdependencias empresariales $(0,29)$, y lleva a que sea factible el pronóstico favorable para la formación del encadenamiento organizacional o alianzas estratégicas, teniendo como unidad básica al caserío para su competitividad.

El indicador descrito aplicado también a la realidad peruana empresarial, y describe una percepción negativa o alcanza una tendencia desfavorable la percepción de los empresarios pesqueros peruanos hacia modalidades de negocios compartidos, es decir desestiman como posibilidad empresarial de realizar alianzas estratégicas: Joint-venture, tradings, franquicias preferentemente; en conclusión, entre otras, sobre la interdependecia empresarial, y la motivación de logro no significativa hacia las alianzas empresariales en empresarios pesqueros peruanos, (Paredes, M. 1997).

Matriz de correlaciones del Coeficiente Tau de Kendall entre la percepción hacia la interdependencia económica y el conocimiento de la demanda de productos.

\begin{tabular}{|l|c|c|c|c|}
\hline \multicolumn{1}{|c|}{ Variable } & Infraestructura & $\begin{array}{c}\text { Procesos del } \\
\text { producto }\end{array}$ & $\begin{array}{c}\text { Percepción } \\
\text { de la oferta }\end{array}$ & $\begin{array}{c}\text { Total } \\
\text { Interdependencia }\end{array}$ \\
\hline Gustos & $-0,17$ & $-0,27 *$ & 0,15 & $-0,16$ \\
\hline Precios & $-0,27 *$ & $-0,15$ & $-0,04$ & $-0,21$ \\
\hline Competencia & $-0,14$ & $-0,18$ & 0,09 & $-0,10$ \\
\hline Posicionamiento & $-0,06$ & 0,02 & $-0,08$ & $-0,08$ \\
\hline Canal & $-0,34 * *$ & $-0,25 *$ & 0,06 & $-0,18$ \\
\hline Oferta & $-0,16$ & $-0,03$ & $0,29 *$ & 0,05 \\
\hline Demanda Total & $-0,14$ & $-0,18$ & 0,20 & $-0,05$ \\
\hline $\begin{array}{l}\mathrm{N}=51 \\
* * \text { significancia }<0,01 \\
* \text { significancia }<0,05\end{array}$ & & & \\
\hline
\end{tabular}

Aun cuando su conocimiento hacia la demanda es muy bajo, se describe positivamente su percepción hacia la interdependencia, y eso lleva a pensar que la formación de cadenas productivas empresariales según la construcción social de cada caserío de la Comunidad se daría por el conocimiento de la oferta. 


\section{Mildred Paredes Tarazona}

Análisis de la bondad de ajuste a la curva normal de la percepción hacia la interdependencia económica y conocimiento de la demanda de productos.

\begin{tabular}{|c|c|c|c|}
\hline Variables (Áreas) & $\mathbf{M}$ & D. E. & Z K-S \\
\hline \multicolumn{4}{|c|}{ Percepción del conocimiento de la demanda } \\
\hline Gusto & 1,14 & 0,30372 & 3,377 \\
\hline Precios & 1,06 & 0,21759 & 3,738 \\
\hline Competencia & 1,14 & 0,3785 & 3,424 \\
\hline Posicionamiento & 1,61 & 0,41082 & 2,443 \\
\hline Canal & 1,55 & 0,23146 & 3,716 \\
\hline Oferta & 1,61 & 0,38518 & 3,623 \\
\hline Demanda total & 8,11 & 1,66381 & 2,591 \\
\hline \multicolumn{4}{|c|}{ Percepción hacia la interdependencia } \\
\hline Infraestructura & 3,59 & 0,31445 & 1,517 \\
\hline Gestión de la producción & 3,395 & 0,30342 & 1,723 \\
\hline Percepción de la oferta & 3,19 & 0,33335 & 3,081 \\
\hline
\end{tabular}

\section{CONCLUSIONES}

1. La articulación económica se construirá o formará entre caserío y caserío, siendo el caserío la variable que mayor discrimina la construcción de la representación social organizacional de la Comunidad Campesina.

2. El objetivo que orienta dicha articulación será prioritariamente el conocimiento de la oferta, más que otros factores como son los procesos productivos.

3. Difiere significativamente la percepción sobre las condiciones de trabajo según el caserío, diferenciándose de caserío en caserío, no así la percepción hacia el medio ambiente. 
El caserío es la unidad productiva en la articulación organizacional de zonas rurales alto andinas, Callejón de Huaylas, Ancash

\section{BIBLIOGRAFÍA}

Alejo Riveros, Argemiro; Rojas Arias, Patricia; Pérez Acosta, Andrés M. 2008, Psicología y asuntos económicos: Una aproximación al estado del arte. Colombia, Internacional Journal of Psychological Research ISSN 2011-7921 Vol. 1 N. ${ }^{\circ}$ 1, pp. 49-57.

Corbera, José. 2008, Tesis de Maestría sustentada el 18 de noviembre de 2008. UNMSM, UPG de la Facultad de Administración.

Holahan, Charles J. 1996, Psicología ambiental, un enfoque general. México, LIMUSA.

Katona, George. 1963, Análisis psicológico del comportamiento económico. Madrid, Ediciones RIALP, S.A.

Paredes, M.; Hernández, H.; Vicuña, L; De Miguel, J. Ma y colaboradores: 2007, Percepción del conocimiento de la demanda de productos y percepción hacia la interdependencia económica en el Caserío Queral, Caraz, Ancash. Vol. 10, N. ${ }^{\circ} 1$ Revista de Investigación en Psicología, IIPs UNMSM.

Paredes, M., De Miguel, J., Hernández, H., Vicuña, L., et al., 2005. El papel de la construcción social de la organización con respecto a la percepción de las condiciones de trabajo y el manejo del medio ambiente en cuatro caseríos de una comunidad campesina andina: Caraz, Ancash, en Revista del Instituto de Investigación Facultad de Ingeniería Geológica, Minera, Metalúrgica y Geográfica, UNMSM, Vol. 8, N. ${ }^{\circ} 16$.

Paredes M.; Jesús María de Miguel Calvo; María José Carvajal Iturry y Miriam Laguna Sánchez 2004, "Construcción Social de la Comunidad para el desarrollo del Callejón de Huaylas, Caraz" Revista de Investigación en Psicología, Instituto de Investigaciones Psicológicas, Facultad de Psicología. UNMSM. Vol 7, N. ${ }^{\circ}$ 1. Lima, julio de 2004.

Paredes Tarazona, Mildred 1997, La interdependencia empresarial factor de calidad en la gestión- caso sector empresarial pesquero peruano. Tesis de Magíster, Universidad Nacional Mayor de San Marcos, Escuela de Postgrado, Unidad de Postgrado de la Facultad de Ciencias Administrativas, Lima. 


\section{Mildred Paredes Tarazona}

Popper, Karl R. 1997. El cuerpo y la mente, Barcelona, Ediciones Paidós I.C.E. de la Universidad Autónoma de Barcelona.

Rubinstein, J.L. 1967. Principios de psicología general. Enciclopedia de psicología, Editorial Grijalbo, México.

Titze, Michael, 1983. Fundamentos del teleoanálisis adleriano, Barcelona, Editorial Helder.

Trivelli, C; Von Hesse, M; Diez, A. y Del Castillo, L. 2000. Desafíos del desarrollo rural en el Perú. Lima, CIES. 
El caserío es la unidad productiva en la articulación organizacional de zonas rurales alto andinas, Callejón de Huaylas, Ancash

\section{Anexo N. ${ }^{\circ} 1$}

Ing. Gaudencio Laureano Valentín (Lima, Mayo 2007): "Identificacion de las condiciones geológicas y ambientales del territorio de la C.C. Cruz de Mayo - Caraz - Ancash"; Documento inédito del Proyecto 2007 - CSI-UNMSM Facultad de Psicología y Facultad de Ingeniería Geológica, Minera Metalúrgica y Geográfica: "Percepción del conocimiento de las expresiones culturales y su revalorización integral en dos comunidades altoandinas en Ancash y Pasco".

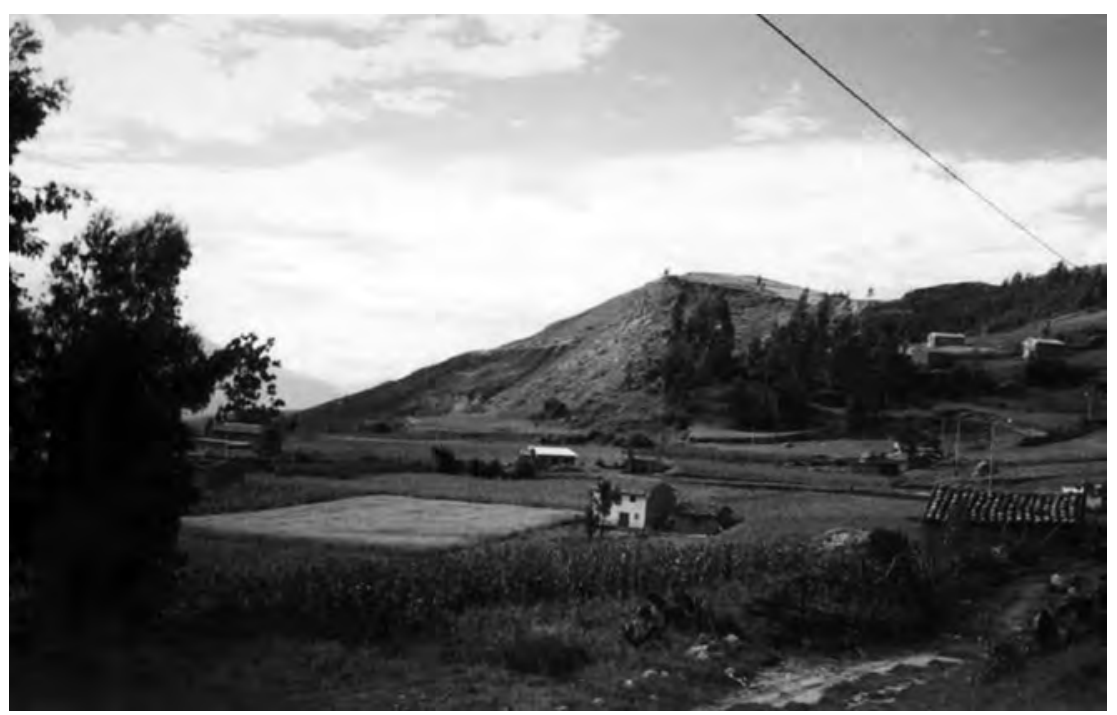

Figura N. ${ }^{\circ}$ 1. Anexo Queral de la C.C. Cruz de Mayo.

Ubicación y accesibilidad

El área de estudio se encuentra en los anexos Miramar, Queral y Pampacocha de la Comunidad Campesina Santa Cruz de Mayo, Provincia Caraz, Región Ancash. El territorio está comprendido dentro del Callejón de Huaylas.

Coordenadas: $9.067^{\circ} \mathrm{S}, 77.817^{\circ} \mathrm{W}$. 


\section{Mildred Paredes Tarazona}

Vías de acceso:

Terrestre:

Lima - Caraz 667 km. por la carretera Panamericana Norte.

Caraz - Queral (Anexo) aproximadamente $35 \mathrm{~km}$ por una trocha angosta.

Aérea:

Lima - Anta

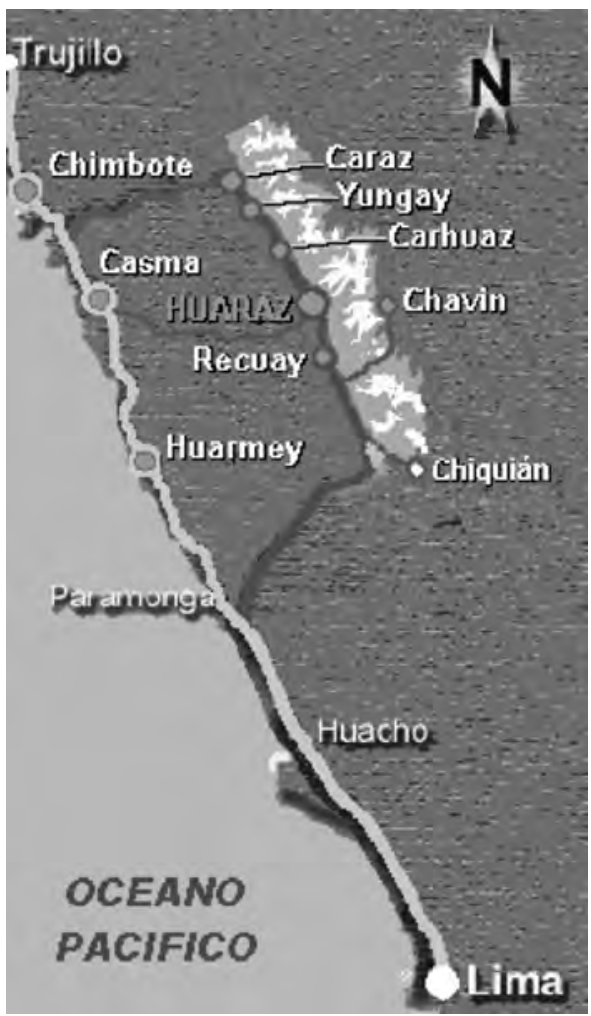

Plano de ubicacion de Caraz. 
El caserío es la unidad productiva en la articulación organizacional de zonas rurales alto andinas, Callejón de Huaylas, Ancash

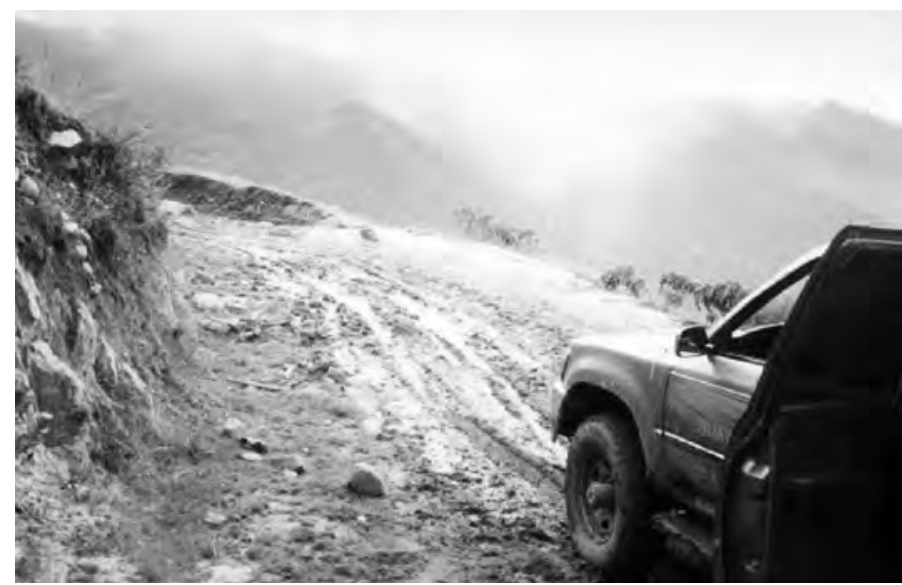

Figura N. ${ }^{\circ}$ 2. Trocha que conduce a Queral.

Altitud:

2285 msnm (Caraz)

3200 msnm (Anexo Miramar)

3500 msnm (Anexo Queral)

3800 msnm (Anexo Pampacocha)

Clima: El clima de la zona estudiada está definida por la geografía regional del Callejón de Huaylas generándose microclimas en espacios cercanos y limitados. Estos climas son:

- Yunga fluvial: clima templado-cálido y relativamente seco comprendido entre Caraz (2285 msnm) y cerca al Anexo Miramar (3200 msnm) con temperaturas cálidas en el día y frío en la noche, con neblinas invernales. Temperatura: Max. $24^{\circ} \mathrm{C}$ y Min. $15^{\circ} \mathrm{C}$. Las precipitaciones no son abundantes.

- Quechua: Clima templado con temperatura Máx. $22^{\circ} \mathrm{C}$ y Min. $12^{\circ} \mathrm{C}$, escasa humedad atmosférica, brisas de valle que soplan en el día desde las quebradas 


\section{Mildred Paredes Tarazona}

estrechas hacia las montañas y durante las noches las brisas de montañas desde las cimas hasta los valles. Con precipitaciones en mayor cantidad que los de la Región Yunga. El anexo de Miramar hasta el anexo Queral presenta este tipo de clima.

- Suni: Clima templado frío, con heladas y precipitaciones mayores que se manifiesta entre Queral y Pampacocha. 
El caserío es la unidad productiva en la articulación organizacional de zonas rurales alto andinas, Callejón de Huaylas, Ancash

\section{Anexo N. $^{\circ} 2$}

Hidrología e hidrogeología

Los recursos hídricos en los anexos Miramar, Queral y Pampacocha de la Comunidad Campesina de Santa Cruz de Mayo provienen de fuentes superficiales y subterráneas.

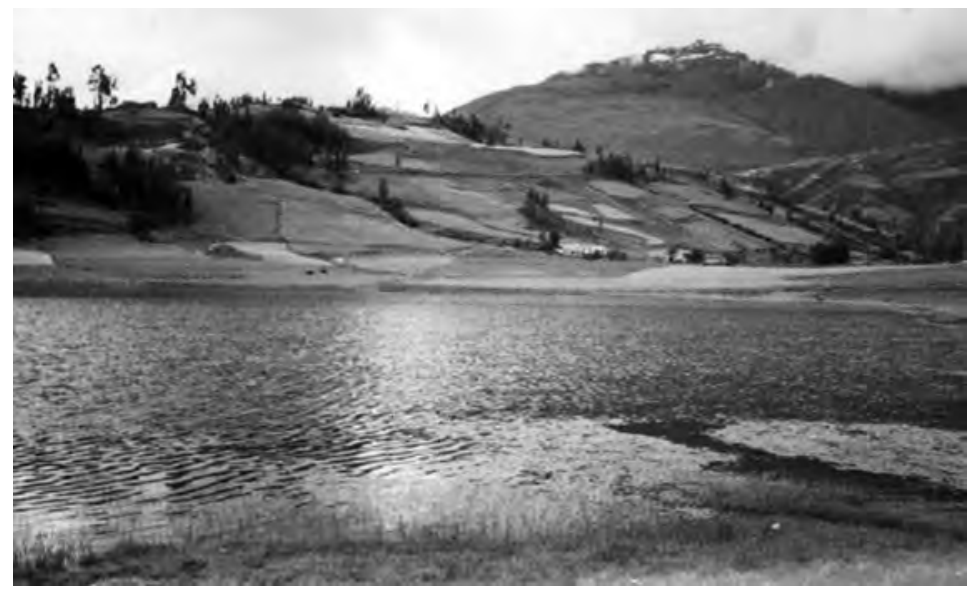

Figura N. ${ }^{\circ}$ 3. Lagunilla de Miramar.

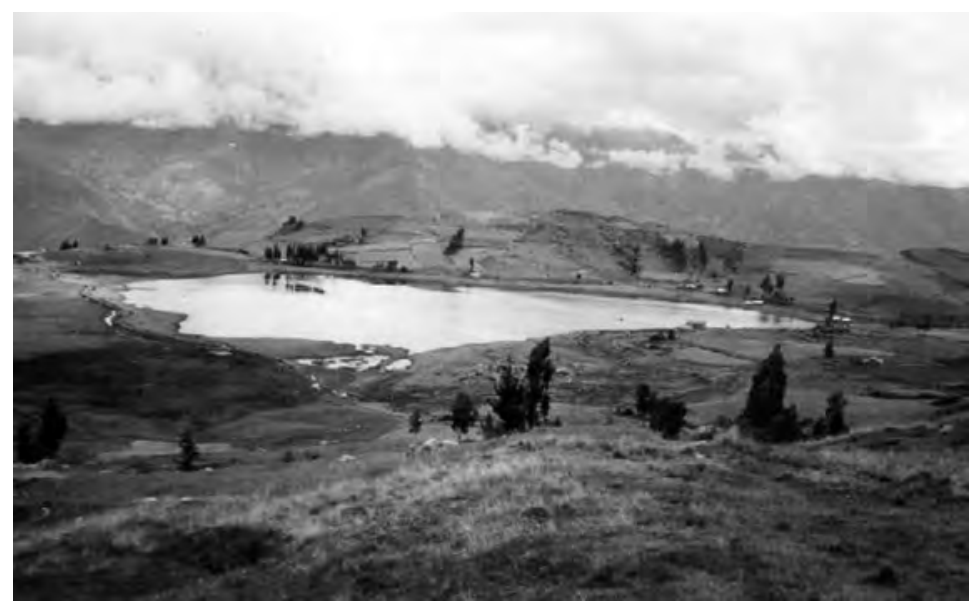

Figura N. ${ }^{\circ}$. Lagunilla de Pampacocha. 


\section{Mildred Paredes Tarazona}

\section{ANEXO N. ${ }^{\circ} 3$ \\ Geodinámica interna y externa de la zona de Caraz}

Geodinámica interna o de origen geológico: la confluencia de las placas convergentes de Nazca y la Sudamérica originan sismos en el Perú y especialmente en Ancash, del cual forma parte Caraz, y los territorios de la Comunidad Campesina Cruz de Mayo. Los sismos se suceden con cierta frecuencia. Por ejemplo, el sismo de 1970 produjo grandes destrozos en el Callejón de Huaylas. Los sismos en esta zona han generado aludes, aluviones, deslizamientos, desprendimientos, agrietamientos, asentamientos, represamientos de ríos, afloramiento de agua subterránea e inundaciones, de allí se concluye que la zona de Caraz es de alta sismicidad.

Geodinámica externa u origen geológico/climático. Se trata de una variedad de problemas que se relacionan con la alteración de la superficie del territorio que, teniendo un origen geológico, son incentivados por factores climáticos (como lluvias), o que, teniendo un origen climático (como cambios inesperados en la temperatura), ocasionan alteraciones en elementos de la geología local, pudiendo, en todo caso, desencadenar una diversidad de acontecimientos y producir daños considerables, en un ambiente de evolución de la Cordillera de los Andes.

En Queral y Miramar encontramos movimientos de tierras que se manifiestan en agrietamientos, desprendimientos y deslizamientos debido a los siguientes factores:

- Clima: las precipitaciones menores de la zona urbana de Caraz se incrementan más según crece la altitud. En Pampacocha, ubicado aproximadamente a más de 3700 msnm, las precipitaciones pluviales son mayores. La variación de la temperatura va de $24^{\circ} \mathrm{C}$ en el día a $0^{\circ} \mathrm{C}$ en la noche. Además, en Queral, Miramar y Pampacocha la cobertura de vegetación para sostenibilidad de suelos es escasa (árboles y arbustos). Estas condiciones climáticas propician el agrietamiento, desprendimiento y deslizamientos de suelos. 
El caserío es la unidad productiva en la articulación organizacional de zonas rurales alto andinas, Callejón de Huaylas, Ancash

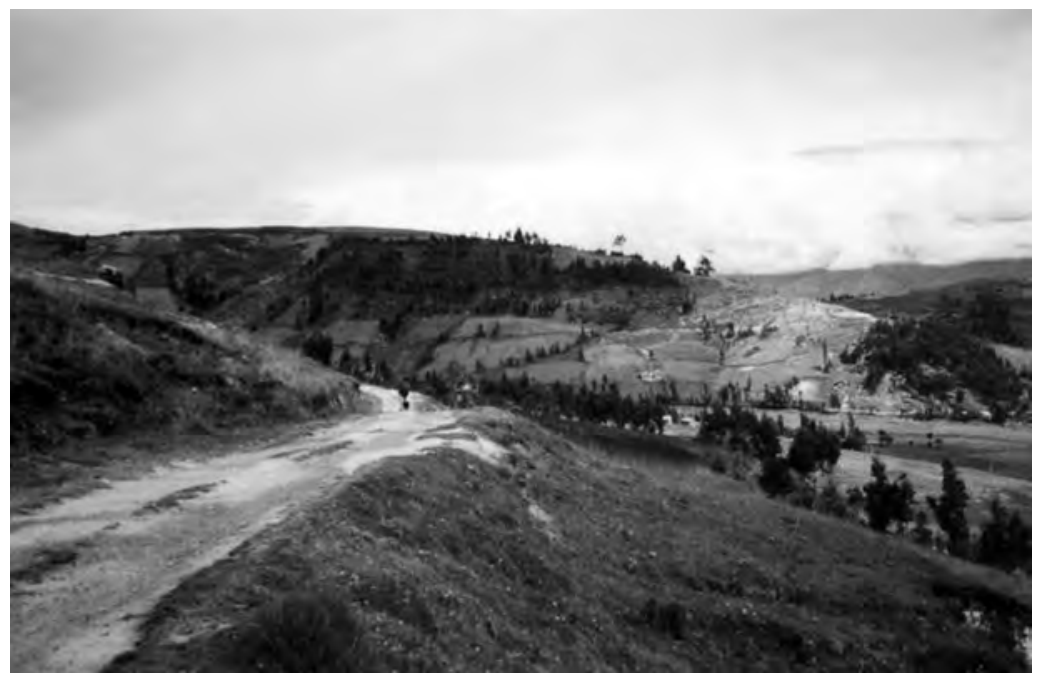

Figura N. ${ }^{\circ}$ 5. Subcuenca de Paron con pocos árboles de eucalipto.

- Pendiente: las laderas de la zona de Miramar alcanzan una inclinación de más de $70^{\circ}$, por lo que estos terrenos son peligrosos por el incremento de tensiones generado por la gravedad y la pendiente.

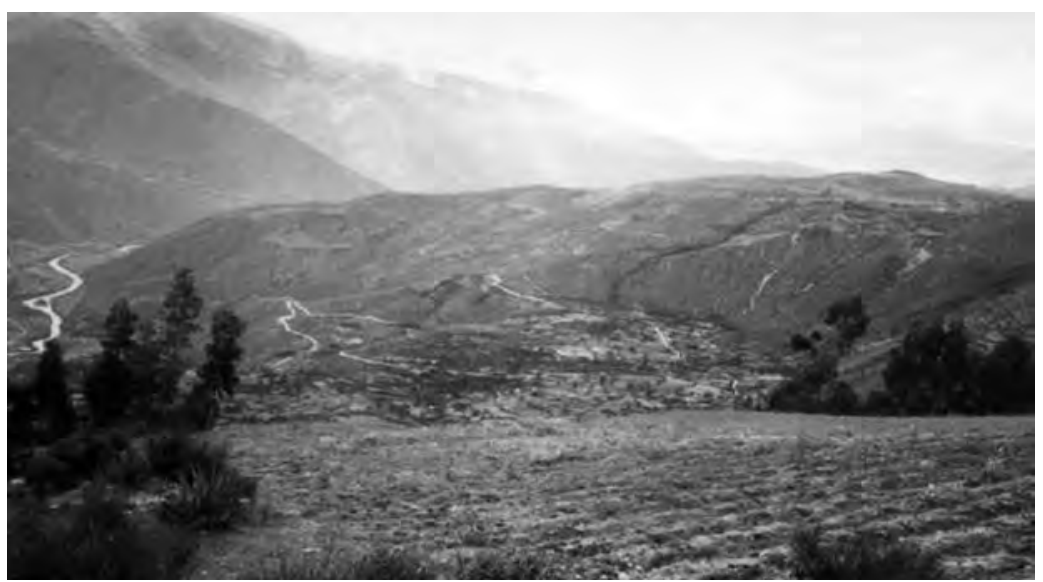

Figura N. ${ }^{\circ}$ 6. Laderas de pendientes fuertes que circundan el área de Paty. 


\section{Mildred Paredes Tarazona}

- Tipo de suelo: el suelo de Miramar y Queral es arenarcillosa con mayor concentración de material arcillosa en muchos sectores de este terreno como podemos observar en el corte de la trocha en la Foto $\mathrm{N}{ }^{\circ} 7$ y también en las fallas que muestra la foto $\mathrm{N}$. 8. El material arcilloso tiene la propiedad de absorber gran cantidad de agua proveniente de las precipitaciones pluviosas y las infiltraciones de la napa freática, pero al crecer la temperatura se va secando hasta llegar a agrietarse, generándose esfuerzos de compresión y de tensión, que finalmente propicia la inestabilidad del suelo. Además, el material de arena que compone este suelo lo torna poroso. Estos espacios generan inestabilidad por estar poco compactados o ser poco consolidados.

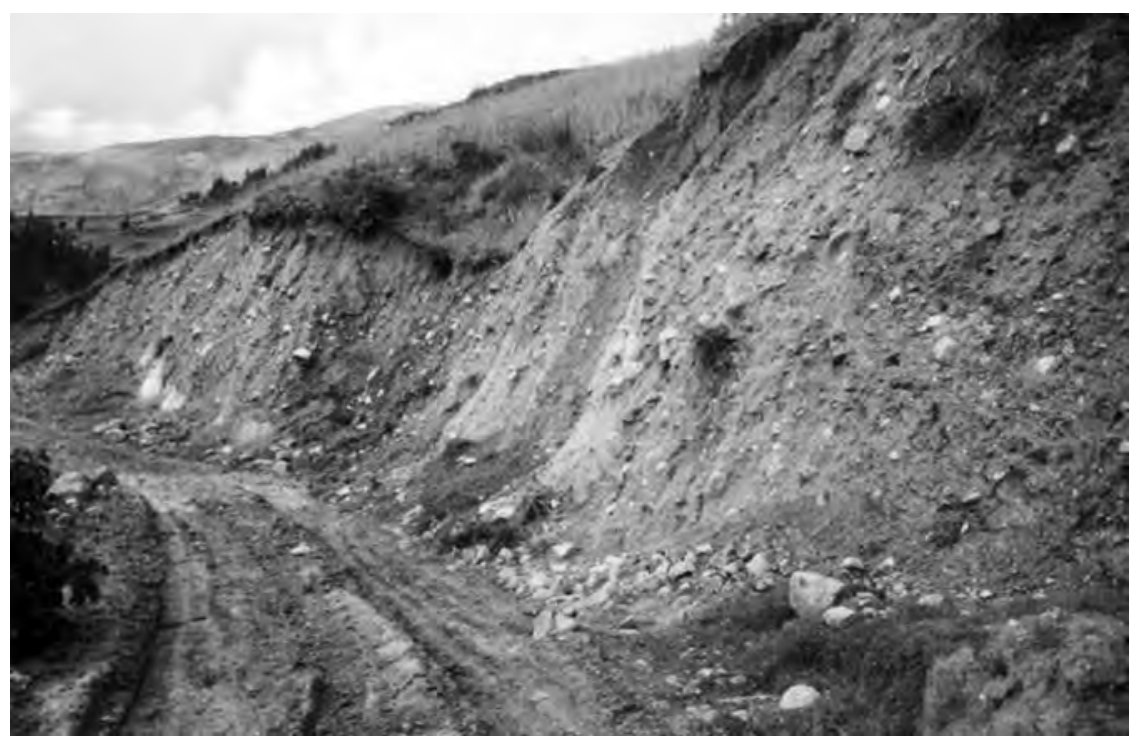

Figura $\mathbf{N}^{\circ}$ 7. Desprendimiento de material arcilloso sobre la trocha.

- Factor hídrico: la filtración de agua (que proviene de las lluvias, lagunas, riachuelos, glaciar) en el suelo poroso produce movimientos de las partículas de este suelo siguiendo la dirección del flujo de agua. A mayor volumen de agua que sature este tipo de suelos puede generar deslizamientos de tierras que pueden resultar peligrosos para los que habitan en las zonas bajas y para los que viven sobre estos terrenos. 
El caserío es la unidad productiva en la articulación organizacional de zonas rurales alto andinas, Callejón de Huaylas, Ancash

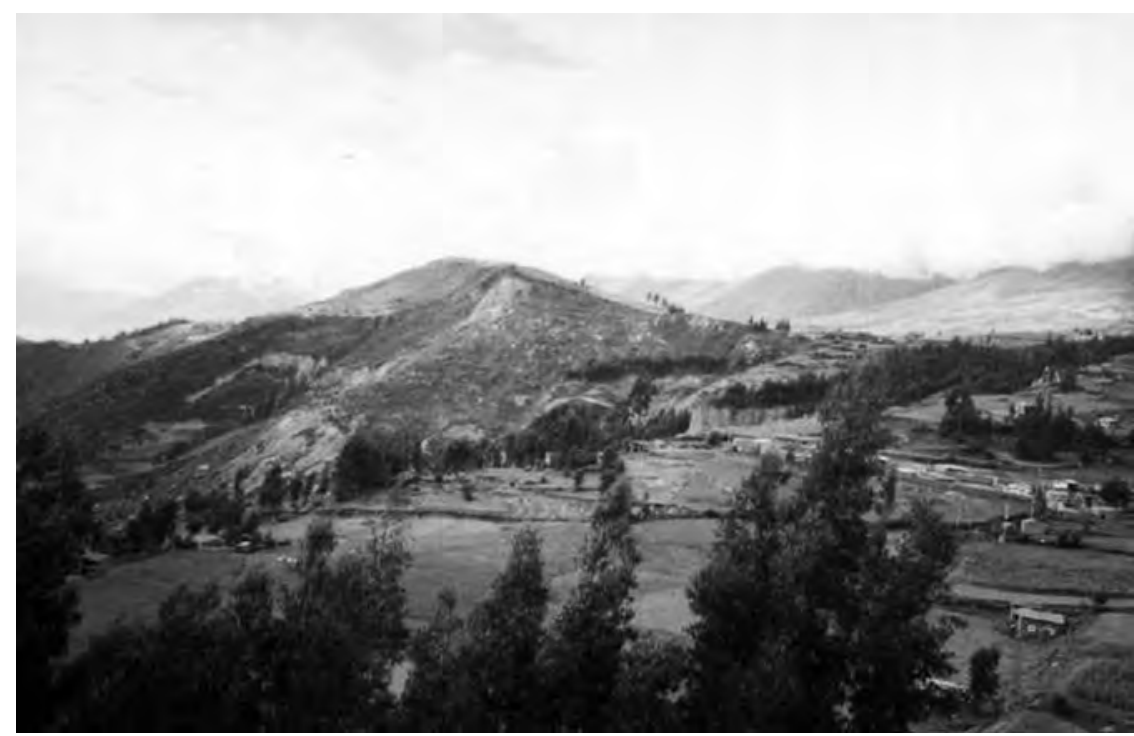

Figura N. ${ }^{\circ}$ 8. Deslizamiento de tierras en la zona de Queral.

Calidad ambiental de la Comunidad Campesina de Santa Cruz de Mayo. Los problemas de contaminación ambiental en el área de la Comunidad se pueden analizar categorizando los problemas del siguiente modo:

- Primera categoría: En el hogar y el trabajo. Las costumbres foráneas están influyendo negativamente en el hogar y en el trabajo. Se puede comprobar que las familias están consumiendo productos industriales como galletas, chocolates, gaseosas, fertilizantes, abonos, etc., cuyos residuos de envolturas y envases los dejan en sus casas o en las chacras. Las familias cocinan con leña con lo que se contamina el aire.

- Segunda categoría: Vecinal o barrial. Se observa que las escuelas y colegios se encuentran abandonados sin servicios de limpieza. El ambiente escolar está contaminado, lo que pone en peligro la salud de los estudiantes y docentes.

- Tercera categoría: Altos niveles de contaminación. La falta de servicios higiénicos y de la red de desagüe contaminan los suelos y el agua. Se lava las ropas en 


\section{Mildred Paredes Tarazona}

los canales de agua que son contaminados por detergentes. La presencia de más de veinte empresas mineras que circundan Caraz impactan también sobre la C.C. Cruz de Mayo. Las actividades mineras contaminan el aire, el suelo y el agua aun cuando están alejados del territorio de esta comunidad. Las brisas de valle que soplan en el día desde las quebradas estrechas hacia las montañas traen en suspensión materiales particulados de tierra y metales a Queral y Pampacocha, y durante las noches las brisas de montañas desde las cimas hasta los valles transportan estos materiales a las zonas más bajas como Miramar. Posteriormente es necesario realizar un estudio más detallado con instrumentos de control de la calidad ambiental del aire, para tener datos más precisos. A esto se agrega la quema de pastizales y residuos sólidos que contaminan el aire.

- Cuarta categoría: Extensión de la urbanización. La construcción de viviendas precarias en lugares de alto riesgo como las viviendas de Miramar cerca de una lagunilla al igual que en Pamapacocha. Incluso es un riesgo para la salud de los niños. Cuentan los comuneros que en la laguna de Pampacocha hubo en cierto tiempo truchas, que luego por algún motivo que desconocen se murieron.

- Quinta categoría: Categoría mundial. El calentamiento global de la Tierra está generando que los glaciares desaparezcan de la Cordillera Blanca. Los glaciares son particularmente importantes, primero, por los recursos hídricos que brindan a los poblados próximos, y además a nivel científico. Los glaciares constituyen las reservas sólidas de agua dulce y por su gran sensibilidad al cambio climático, los glaciares tropicales representan excelentes indicadores de la evolución del clima. Por lo tanto, la reducción de las áreas de nieve está ocasionando la pérdida de las reservas sólidas de agua en esta zona. 